\title{
Fractionation of Organic Carbon and Stock Measurement in the Sundarbans Mangrove Soils of Bangladesh
}

\author{
Sayada Momotaz Akther ${ }^{1}$, Md Mahfuz Islam ${ }^{1,2 *}$, Md Faruque Hossain ${ }^{3}$, Zakia Parveen ${ }^{1}$ \\ ${ }^{1}$ Department of Soil, Water and Environment, University of Dhaka, Dhaka, Bangladesh \\ ${ }^{2}$ Department of Crop and Soil Sciences, North Carolina State University, Raleigh, NC, USA \\ ${ }^{3}$ OSCM, FBA, American International University-Bangladesh, Kuril, Dhaka, Bangladesh \\ Email: momotazenadu789@gmail.com, *mislam25@ncsu.edu, *mdmahfuz789@gmail.com, \\ mfhossain@aiub.edu,zakiaparveen56@du.ac.bd
}

How to cite this paper: Akther, S. M., Islam, M. M., Hossain, M. F., \& Parveen, Z. (2021). Fractionation of Organic Carbon and Stock Measurement in the Sundarbans Mangrove Soils of Bangladesh. American Journal of Climate Change, 10, 561-580. https://doi.org/10.4236/ajcc.2021.104028

Received: August 3, 2021

Accepted: December 28, 2021

Published: December 31, 2021

Copyright $\odot 2021$ by author(s) and Scientific Research Publishing Inc. This work is licensed under the Creative Commons Attribution International License (CC BY 4.0).

http://creativecommons.org/licenses/by/4.0/

(c) (i) Open Access

\begin{abstract}
Mangrove soils are well known for their high capacity of storing organic carbon (SOC) in various pools; however, a relatively small change in SOC pools could cause significant impacts on greenhouse gas concentrations. Thus, for an in-depth understanding of SOC distribution and stock to predict the role of Sundarbans mangrove in mitigating global warming and greenhouse effects, different extraction methods were employed to fractionate the SOC of Sundarbans soils into cold-water (CWSC) and hot-water (HWSC) soluble, moderately labile (MLF), microbial biomass carbon (MBC), and resistant fractions (RF) using a newly developed modified-method. A significant variation in total SOC $(\mathrm{p}<0.001)$, SOC stock $(\mathrm{p}<0.001)$ and soil bulk density $(\mathrm{p}<$ $0.05)$ at the Sundarbans mangrove forest were observed. In most soils, bulk density increased from the surface to $100 \mathrm{~cm}$ depth. The total SOC concentrations were higher in most surface soils and ranged from $1.21 \% \pm 0.02 \%$ to $8.19 \% \pm 0.09 \%$. However, $\mathrm{C}$ in lower layers may be more resistant than that of upper soils because of differences in compositions, sources and environmental conditions. SOC was predominately associated with the resistant fraction ( $81 \%$ - 97\%), followed by MLF ( $2 \%-10 \%)$, HWSC (1\% - 4\%), MBC ( 0\% $4 \%)$, and CWSC $(\sim 0 \%-3 \%)$. The significant positive correlations between different $\mathrm{C}$ fractions suggested that $\mathrm{C}$ pools are interdependent and need proper management plans to increase these pools in Sundarbans soils. The SOC stock of the studied areas ranged between $16.75 \pm 3.83$ to $135.12 \pm 28.61$ $\mathrm{kg} \cdot \mathrm{C} \cdot \mathrm{m}^{-2}$ in $1 \mathrm{~m}$ soil profile and has an average of $31.80 \mathrm{~kg} \cdot \mathrm{C} \cdot \mathrm{m}^{-2}$. The substratum soils had more carbon than the upper layers in the Sundarbans wetland due to burial and preservation of carbon by frequent tidal inundation. A
\end{abstract}


higher SOC stock in the soil profile and its primary association in resistant fractions suggested that Sundarbans mangrove soil is sequestering carbon and thereby serving as a significant carbon sink in Bangladesh.

\section{Keywords}

Carbon Sequestration, Carbon Sink, Carbon Source, Soil Organic Carbon Stock, Sundarbans Mangrove Forest

\section{Introduction}

Soil organic carbon storage, resulting from a range of natural biogeochemical processes, is a major ecosystem service that regulates the chemical, physical and biological properties of soil. The amount of organic $\mathrm{C}$ in soil (1500 GT) represents approximately $50 \%$ of the total terrestrial carbon $(3060 \mathrm{GT})$, and this pool is approximately two times of the atmospheric pool of 760 GT (Oelkers \& Cole, 2008; Lal, 2008; Hossain et al., 2007). Only the ocean has a larger C pool ( 38,400 GT), although most are inorganic forms (Houghton, 2007). Moreover, it is estimated that $\sim 20 \%-30 \%$ of terrestrial SOCs are stored in wetlands (Bridgham et al., 2006), although only $5 \%-8 \%$ of land surfaces consist of wetlands (Mitsch et al., 2013). Therefore, wetland soils are the major carbon sinks on earth because of higher amounts of stored organic carbon.

Mangrove plays a crucial role worldwide with a multitude of services, including carbon and nutrients cycling and coastal protection (Koshiba et al., 2013; Alongi, 2008). Several studies identified mangroves are most carbon-rich among the forest ecosystems (Eid et al., 2019; Murdiyarso et al., 2015; Donato et al., 2011; Kauffman et al., 2011) and act as a powerful atmospheric C sink (three times the biomass) because of their higher primary production capacity (Twilley et al., 1992). Although mangroves are efficient in sequestering and conserving $C$ in soils, sediments, and plant biomass, there are concerns that global warming may release carbon into the atmosphere as $\mathrm{CO}_{2}$, the major greenhouse gas (Eid et al., 2019; Alongi, 2012). Still, situations may vary from place to place and time to time (Hossain et al., 2020).

Sundarbans plays a crucial but underrated role in carbon storage and greenhouse gas regulation. Sundarbans mangrove is currently experiencing the highest rates of degradation among existing forest ecosystems from both natural including cyclone, storm surge, lightning, pest and diseases and anthropogenic disturbances like deforestation, pollution, urban and coastal development, agriculture and aquaculture conversion, hydrological disruptions, and over-exploitation of timber, wood, and fish (Grellier et al., 2017; Spaulding et al., 2010; Alongi, 2012; Duke et al., 2007; Alongi, 2002). Besides the loss of above-ground carbon by mangrove disturbance, significant $\mathrm{CO}_{2}$ is released into the atmosphere due to the decomposition of SOC. This wetland provides a potential sink for atmos- 
pheric carbon, but it becomes a carbon source and accelerates climate change if not appropriately managed. So far, limited studies have been done to assess its roles and potentials in carbon sequestration. The Sundarbans mangrove is attracting increasing interest in climate change mitigation strategies because of their vulnerabilities to land-use changes, presence of large $\mathrm{C}$ stocks and several other ecosystem services (Hossain et al., 2020; Murdiyarso et al., 2009).

Soil organic carbon has been an essential topic of study in the field of soil and environmental sciences. Simply, estimating total SOC in only surface soils is insufficient to study the storage, stability, and dynamics of SOC concerning ecosystem carbon balance. Because the SOC pool is very dynamic from the surface layer to $1 \mathrm{~m}$ depth (Hossain et al., 2020; Lal, 2008) and the labile pool has a lower turnover time ( $<5$ years) compared to resistant residues (20 - 40 years), which are physically or chemically protected (Hoyle \& Murphy, 2006). Moreover, the stability of SOC is regulated by various biotic and abiotic factors in soil coupled with a variety of biogeochemical processes. Therefore, it is vital to characterize SOC quality relating to biodegradability. It has been recommended that changes in the different SOC fractions indicate alterations in soil use than total soil organic carbon content. The SOC fractionation and relation of different fractions with soil properties can generate the theoretical basis for adopting sustainable land management approaches (Pinheiro et al., 2004).

To develop proper soil management strategies in the Sundarbans area, it is necessary to determine SOC storage and its pool. Because of uncertainties and lack of consensus regarding the purpose and use of soil carbon storage in Sundarbans inventories, the information available is too fragmented to use on a broader scale. Considering all these factors, several studies were combined from the literature and developed a new chemical fractionation method to separate the SOC into various components based on the solubility in numerous extracting agents and their dynamics in the ecosystem of Sundarbans. This study also aims to measure SOC storage in $1 \mathrm{~m}$ depth of Sundarbans mangrove soil.

\section{Materials and Methods}

\subsection{Description of the Study Area}

The world's largest single-block mangrove Sundarbans is located on the GangesBrahmaputra Delta, where it joins the Bay of Bengal, southwestern part of Bangladesh $\left(21^{\circ} 30^{\prime} \mathrm{N}-22^{\circ} 30^{\prime} \mathrm{N}\right.$ and $\left.89^{\circ} 00^{\prime} \mathrm{E}-89^{\circ} 55^{\prime} \mathrm{E}\right)$. Approximately $60 \%$ of the forest is situated in Bangladesh and the rest is in West Bengal, India (Hussain \& Acharya, 1994). Bangladesh Sundarbans is divided into two forest divisions and four administrative ranges, namely Chandpai, Sarankhola, Buri Guoalini, and Nalian and sixteen different stations. A humid subtropical climate prevails in this area. The average annual temperature, rainfall, and humidity of Sundarbans mangrove forest vary from $17^{\circ} \mathrm{C}-32^{\circ} \mathrm{C}, 1640-2000 \mathrm{~mm}$, and $70 \%-80 \%$, respectively (Siddiqi, 2001). 


\subsection{Soil Sampling and Preparation}

Approximately $3 / 4$ of the terrestrial carbon is present in the upper $1 \mathrm{~m}$ soil (Lal, 2008; Hossain et al., 2007), three consecutive depths $(0-15 \mathrm{~cm}, 15-50 \mathrm{~cm}$, and $50-100 \mathrm{~cm}$ ) of soil profile were selected for soil samples collection. Composite soil samples from ten different locations $\left(22.302777^{\circ} \mathrm{N}, 89.617777^{\circ} \mathrm{E} ; 22.241888^{\circ} \mathrm{N}\right.$, $89.570666^{\circ} \mathrm{E} ; 21.875100^{\circ} \mathrm{N}, 89.836111^{\circ} \mathrm{E} ; 21.845278^{\circ} \mathrm{N}, 89.795277^{\circ} \mathrm{E} ; 21.816111^{\circ} \mathrm{N}$, $89.457777^{\circ} \mathrm{E} ; 21.771017^{\circ} \mathrm{N}, 89.551863^{\circ} \mathrm{E}, 21.858611^{\circ} \mathrm{N}, 89.768611^{\circ} \mathrm{E} ; 22.129722^{\circ} \mathrm{N}$, $89.712500^{\circ} \mathrm{E} ; 22.427500^{\circ} \mathrm{N}, 89.592500^{\circ} \mathrm{E}$ and $22.322373^{\circ} \mathrm{N}, 89.431939^{\circ} \mathrm{E}$ ) of the

Sundarbans mangrove forest were collected (Figure 1). Collected samples were

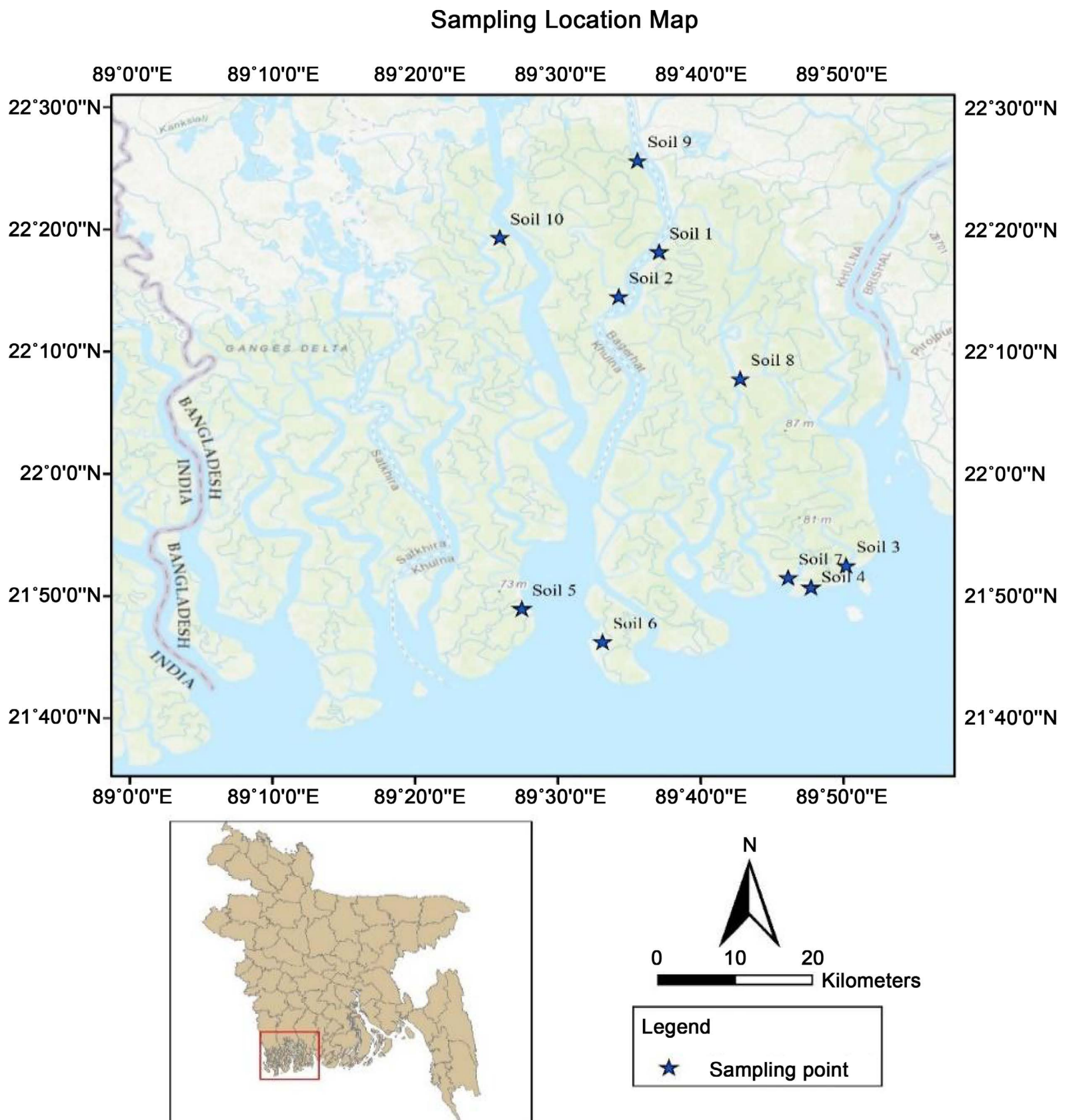

Figure 1. Sampling sites of the study area in Sundarbans. 
placed in separate polythene bags, labeled, and brought into the laboratory to analyze different parameters. For bulk density determination, core samples from $0-15 \mathrm{~cm}, 15-50 \mathrm{~cm}$ and $50-100 \mathrm{~cm}$ were collected by a locally produced peat auger. This auger features an open-faced $10.2 \mathrm{~cm}$ diameter cylinder that efficiently collects relatively undisturbed wetland soils (Donato et al., 2011). The samples were air-dried spreading on clean polythene sheets. Visible roots and debris were carefully discarded from soils. Massive aggregates of resulting dried samples were broken with a wooden hammer. Ground soils were screened using a $2 \mathrm{~mm}$ stainless steel sieve and preserved properly for analysis.

\subsection{Analytical Procedure}

Soil Physical properties viz bulk density (Blake, 1965), texture (Bouyoucos, 1936), soil acidity $(\mathrm{pH})$ (Soil: Water $=1: 2.5, \mathrm{w} / \mathrm{v}$ ), and redox potential $(\mathrm{Eh})$ (Soil: Water $=1: 5, \mathrm{w} / \mathrm{v}$ ) were determined. Organic carbon was determined by Walkley and Black's wet oxidation method (Walkley \& Black, 1934). A new sequential fractionation scheme was developed for the fractionation of SOC in Sundarbans soils based on Erich et al. (2012) and Ghani et al. (2003) with modification (Table 1). The SOC is usually present in soils as labile, moderately labile, microbial biomass, and resistant fractions. Two major parts of the labile $\mathrm{C}$ pool were usually studied: Cold-Water soluble carbon (CWSC) and Hot-Water soluble carbon (HWSC) (Ghani et al., 2003). Quantitatively the first one is linked to dissolved organic carbon. The second one contains more stable elements that form nutrients and energy reservoirs for plants and microorganisms. Labile $\mathrm{C}$ pool is a critical $\mathrm{CO}_{2}$ source and directly changes soil $\mathrm{CO}_{2}$ flow because of high biodegradation (Gregorich et al., 2003). Moderately labile SOC is a reservoir of

Table 1. Extraction methods for different fractions of SOC in Sundarbans soils.

\begin{tabular}{|c|c|c|c|c|c|}
\hline Fractions & $\begin{array}{l}\text { Extract } \\
\text { solution }\end{array}$ & $\begin{array}{l}\text { Soil } \\
\text { (g) }\end{array}$ & $\begin{array}{l}\text { Solution } \\
\quad(\mathrm{ml})\end{array}$ & Conditions & References \\
\hline $\begin{array}{l}\text { Cold-Water } \\
\text { soluble C } \\
\text { (CWSC) }\end{array}$ & $\begin{array}{l}\text { Distilled } \\
\text { water }\end{array}$ & 3 & 30 & $\begin{array}{l}30 \mathrm{~min} \text { extraction at } 20^{\circ} \mathrm{C} \\
\text { and } 20 \mathrm{~min} \text { centrifugation } \\
\text { at } 3000 \mathrm{rpm}\end{array}$ & $\begin{array}{l}\text { Ghani et al., } \\
2003\end{array}$ \\
\hline $\begin{array}{l}\text { Hot-Water } \\
\text { soluble C } \\
\text { (HWSC) }\end{array}$ & $\begin{array}{l}\text { Distilled } \\
\text { water }\end{array}$ & 3 & 30 & $\begin{array}{l}16 \mathrm{hrs} \text { extraction at } 80^{\circ} \mathrm{C} \text { and } \\
20 \mathrm{~min} \text { rotation at } 3000 \mathrm{rpm}\end{array}$ & $\begin{array}{l}\text { Ghani et al., } \\
2003\end{array}$ \\
\hline $\begin{array}{l}\text { Moderately } \\
\text { Labile C } \\
(\mathrm{MLF})\end{array}$ & $\begin{array}{l}125 \mathrm{mM} \\
\mathrm{Na}_{4} \mathrm{P}_{2} \mathrm{O}_{7} \\
(\mathrm{pH} \mathrm{5})\end{array}$ & 3 & 25 & $\begin{array}{l}24 \mathrm{hrs} \text { end-over-end rotation } \\
(40 \mathrm{rpm}) \text { at } 25^{\circ} \mathrm{C}\end{array}$ & $\begin{array}{l}\text { Erich et al., } \\
2012\end{array}$ \\
\hline $\begin{array}{l}\text { Microbial } \\
\text { Biomass C } \\
(\mathrm{MBC})\end{array}$ & $\begin{array}{l}0.5 \mathrm{M} \\
\mathrm{K}_{2} \mathrm{SO}_{4}\end{array}$ & 20 & 100 & $\begin{array}{l}\text { 1-hour shake, sterilization, } \\
\text { and extraction }\end{array}$ & $\begin{array}{l}\text { Ghani et al., } \\
2003\end{array}$ \\
\hline $\begin{array}{l}\text { Resistant C } \\
\text { Fraction } \\
(\mathrm{RF})\end{array}$ & \multicolumn{4}{|c|}{$\mathrm{RF}=$ Total SOC -} & This Study \\
\hline
\end{tabular}


less decomposable soil organic carbon and its primary and essential function is cation exchange. The organic-mineral aggregates constitute a significant source of this $\mathrm{C}$ pool. Although the microbial biomass carbon (MBC) pools only represent $1 \%-5 \%$ of the total SOC in soil, it is the driving force of the soil carbon cycle (Erich et al., 2012).

\subsubsection{Labile Fraction: Cold-Water (CWSC) and Hot-Water Soluble (HWSC)}

For the CWSC fraction, $3 \mathrm{~g}$ soil of each sample was weighed to $50 \mathrm{ml}$ centrifuge tubes (polypropylene), shaken on an end-over-end shaker and centrifuged as described in Table 1. The supernatants were filtered via $0.45 \mathrm{~mm}$ cellulose-nitrate-membrane filter papers into clean plastic bottles for carbon analysis. For the second fraction, residue soils from the first step were shaken for 10 seconds with $30 \mathrm{ml}$ distilled water on a vortex shaker to suspend soil in the added water and then left for $16 \mathrm{~h}$ in a hot-water bath at $80^{\circ} \mathrm{C}$. After completing the water bath extraction, the shaking was repeated for $10 \mathrm{~s}$ to release HWSC from soil to hot water fully. These tubes were centrifuged and supernatants were filtered as described above. Finally, the organic HWSC was determined by multiplying the total hot-water soluble $\mathrm{C}$ by 0.963 because the average inorganic $\mathrm{C}$ of the soils was $3.7 \%$

\subsubsection{Moderately Labile Fraction (MLF)}

Soil samples were extracted using $125 \mathrm{mM} \mathrm{Na}_{4} \mathrm{P}_{2} \mathrm{O}_{7}$ (pH 5). Pyrophosphate is usually used to extract $\mathrm{Fe}-\mathrm{Al}$ bound soil organic C (Erich et al., 2012; WattelKoekkoek et al., 2001; Schnitzer \& Schuppli, 1989). Thus, this fraction possibly chemically sorbed $\mathrm{C}$ on the soil surface and protected from decay. The extraction step was employed by adding $25 \mathrm{ml}$ of extractants in the $3 \mathrm{~g}$ residue soils of HWSC fraction and set for 24 hours end-over-end rotation at $40 \mathrm{rpm}$. The suspensions were kept in a safe place to settle down overnight. The supernatant was cautiously transferred and filtered by repeating the previously described filters.

\subsubsection{Microbial Biomass Carbon (MBC) Fraction}

A chloroform fumigation-extraction technique was employed to assess microbial biomass C in field-moist soils (Ghani et al., 2003). Field moist soil was used because this soil represents a nearly exact amount of microbial biomass $\mathrm{C}$ (Vance et al., 1987). Five gram soil samples (dry weight basis) were fumigated by chloroform for $24 \mathrm{~h}$ and extracted with $0.5 \mathrm{M} \mathrm{K}_{2} \mathrm{SO}_{4}$ for 2 hours shaking. Following the same procedure, extraction of a similar set of non-fumigated samples was performed. Finally, the MBC fraction was estimated by subtracting non-fumigated soil carbons from fumigated C (Ghani et al., 2003).

\subsubsection{Resistant Fraction (RF)}

The resistant fraction is physicochemically protected against decomposition and measured by subtracting the $\mathrm{C}$ concentrations of the above four fractions from 
the total SOC. The amount of $\mathrm{C}$ in all the extracts was determined by injecting about $15-20 \mathrm{~mL}$ extracts in the detection chamber of the Shimadzu TOC analyzer (Model: 5000A). The injections were carried out three times for some random samples and found $98 \%$ to $99.5 \%$ similarity in the same extracts.

\subsubsection{Soil Organic Carbon (SOC) Stock}

Storages of SOC in Sundarbans soils were determined according to Hossain et al. $(2015,2007)$. The SOC storage is expressed on an oven-dry weight basis and calculated by using three variables, namely horizon thickness, soil bulk density and \% organic carbon (Meersmans et al., 2008). The SOC stock was estimated by the following formula.

$$
\text { SOC storage } \mathrm{kg} \mathrm{C} \mathrm{m}^{-2}=\frac{\% \mathrm{OC} \times \mathrm{BD} \times \text { Thichness of Layer }(\mathrm{cm}) \times 10}{100 \%}
$$

where, OC is organic carbon, BD is soil bulk density $\left(\mathrm{g} \cdot \mathrm{cm}^{-3}\right)$, and 10 is the conversion factor from $\mathrm{g} \cdot \mathrm{cm}^{-2}$ to $\mathrm{kg} \cdot \mathrm{m}^{-2}$.

\subsection{Statistical Analyses}

The data were statistically analyzed using Minitab 2019 and Microsoft excel 2016 versions. Descriptive statistical analysis was used to establish trends and differences of date between variables like mean, median, standard deviation, minimum and maximum and also to produce tables, bars. Inferential statistical analyses were used to test the relationships between different variables under the study. Relationships were assumed as significant when the $\mathrm{p}<0.05$.

\section{Results}

The physical and chemical properties of Sundarbans mangrove soils, namely total organic Carbon (SOC), bulk density, soil acidity $(\mathrm{pH})$, redox potential (Eh), and clay content ranged from $1.114 \% \pm 0.01 \%$ to $8.494 \% \pm 0.03 \%, 0.96$ to 1.74 $\mathrm{g} \cdot \mathrm{cm}^{-3}, 6.12 \pm 0.02$ to $8.1 \pm 0.01,-99 \pm 1.81$ to $26.5 \pm 2.22 \mathrm{mV}$, and $6.63 \%$ to $32.81 \%$, respectively (Table 2 ). There was a significant variation in total SOC $(\mathrm{p}<0.001)$, soil bulk density ( $\mathrm{p}<0.05)$, and SOC stock $(\mathrm{p}<0.001)$ at the Sundarbans Mangrove (Table 3). Soil bulk density increased from surface to $100 \mathrm{~cm}$ depth in most areas. Data in Table 4 and Figure 2 highlight the concentrations of five $\mathrm{C}$ fractions and their percentage distribution that varied in $\mu \mathrm{g} \cdot \mathrm{g}^{-1}$ between $568.8 \pm 48.9$ to $180.8 \pm 32.0(0 \%-3 \%)$ for cold water soluble, $280.0 \pm 11.2$ to $646.7 \pm 17.0(1 \%-4 \%)$ for hot-water soluble, $538.7 \pm 30.1$ to $1515.37 \pm 12.3(2 \%$ to $10 \%)$ for moderately labile, $231.5 \pm 10.2$ to $604.8 \pm 52.4(0 \%-4 \%)$ for microbial biomass and $9187.2 \pm 100$ to $82744.6 \pm 300$ (81\% to $97 \%)$ for the resistant fraction in different depth of Sundarbans soil profile. These five fractions maintained a significant correlation $(\mathrm{p}<0.05)$ between themselves and the studied soil parameters (Table 5). The PCA study showed about $73.5 \%$ of the total variability could be explained, while cluster analysis exhibited $>99 \%$ similarities between ten studied locations (Figure 3 ). Considering soil profile depth $(1 \mathrm{~m})$, 
Table 2. Studied physical and chemical properties of the Sundarbans soils.

\begin{tabular}{|c|c|c|c|c|c|c|c|c|c|c|c|}
\hline Soil & $\begin{array}{c}\text { Depth } \\
(\mathrm{cm})\end{array}$ & $\begin{array}{l}\text { OC } \\
(\%)\end{array}$ & $\begin{array}{c}\mathrm{BD} \\
\left(\mathrm{g} \cdot \mathrm{cm}^{-3}\right)\end{array}$ & $\mathrm{pH}$ & $\begin{array}{c}\mathrm{Eh} \\
(\mathrm{mV})\end{array}$ & Soil & $\begin{array}{c}\text { Depth } \\
(\mathrm{cm})\end{array}$ & $\begin{array}{l}\text { OC } \\
(\%)\end{array}$ & $\begin{array}{c}\mathrm{BD} \\
\left(\mathrm{g} \cdot \mathrm{cm}^{-3}\right)\end{array}$ & $\mathrm{pH}$ & $\begin{array}{c}\mathrm{Eh} \\
(\mathrm{mV})\end{array}$ \\
\hline \multirow{3}{*}{ S1 } & $0-15$ & $1.833 \pm 0.03$ & 1.15 & 7.6 & -79 & \multirow{3}{*}{ S6 } & $0-15$ & $1.326 \pm 0.02$ & 1.28 & 7.4 & -65 \\
\hline & $15-50$ & $1.716 \pm 0.05$ & 1.28 & 8.1 & -90 & & $15-50$ & $1.482 \pm 0.07$ & 1.43 & 7.6 & -73 \\
\hline & $50-100$ & $1.283 \pm 0.04$ & 1.32 & 7.2 & -102 & & $50-100$ & $1.512 \pm 0.04$ & 1.56 & 7.8 & -98 \\
\hline \multirow{3}{*}{ S2 } & $0-15$ & $1.95 \pm 0.04$ & 1.24 & 7.9 & -99 & \multirow{3}{*}{ S7 } & $0-15$ & $1.677 \pm 0.22$ & 1.01 & 7.6 & -79 \\
\hline & $15-50$ & $1.833 \pm 0.02$ & 1.21 & 8 & -97 & & $15-50$ & $1.521 \pm 0.09$ & 1.23 & 8.1 & -69 \\
\hline & $50-100$ & $1.463 \pm 0.03$ & 1.17 & 7.7 & -113 & & $50-100$ & $1.828 \pm 0.02$ & 1.29 & 7.1 & -100 \\
\hline \multirow{3}{*}{ S3 } & $0-15$ & $1.443 \pm 0.03$ & 0.96 & 7.8 & -85 & \multirow{3}{*}{ S8 } & $0-15$ & $1.404 \pm 0.03$ & 1.19 & 7.4 & -83 \\
\hline & $15-50$ & $1.209 \pm 0.02$ & 1.18 & 7.9 & -95 & & $15-50$ & $1.638 \pm 0.05$ & 1.56 & 7.7 & -91 \\
\hline & $50-100$ & $1.587 \pm 0.01$ & 1.22 & 7.3 & -122 & & $50-100$ & $1.264 \pm 0.03$ & 1.51 & 7.3 & -109 \\
\hline \multirow{3}{*}{ S4 } & $0-15$ & $7.020 \pm 0.04$ & 1.36 & 6.1 & 26.5 & \multirow{3}{*}{ S9 } & $0-15$ & $1.482 \pm 0.05$ & 1.14 & 7.3 & -75 \\
\hline & $15-50$ & $8.190 \pm 0.09$ & 1.74 & 6.4 & 2.3 & & $15-50$ & $1.365 \pm 0.04$ & 1.44 & 7.4 & -87 \\
\hline & $50-100$ & $8.494 \pm 0.03$ & 1.67 & 6.2 & -48 & & $50-100$ & $1.114 \pm 0.01$ & 1.51 & 6.8 & -107 \\
\hline \multirow{3}{*}{ S5 } & $0-15$ & $1.755 \pm 0.05$ & 1.25 & 7.5 & -70 & \multirow{3}{*}{$\mathrm{S} 10$} & $0-15$ & $1.638 \pm 0.04$ & 1.15 & 7.3 & -83 \\
\hline & $15-50$ & $1.794 \pm 0.06$ & 1.43 & 7.4 & -74 & & $15-50$ & $1.560 \pm 0.09$ & 1.28 & 7.5 & -90 \\
\hline & $50-100$ & $2.036 \pm 0.02$ & 1.46 & 7.2 & -89 & & $50-100$ & $1.833 \pm 0.03$ & 1.38 & 7.1 & -105 \\
\hline
\end{tabular}

Table 3. Analysis of variance (ANOVA) for different soil properties in ten studied locations.

\begin{tabular}{ccc}
\hline Properties & $\begin{array}{c}\text { Value Range }(\mathrm{n}=9 \times 10,9 \text { from each location } \\
\text { and } 3 \text { from each layer })\end{array}$ & ANOVA \\
\hline Total Organic Carbon & $1.114 \% \pm 0.01 \%-8.494 \% \pm 0.03 \%$ & $\mathrm{p}<0.001, \mathrm{~F}=200.21, \mathrm{Fcrit}=2.21$ \\
SOC Stock & $16.75 \pm 3.83-135.12 \pm 28.61 \mathrm{~kg} \cdot \mathrm{C} \cdot \mathrm{m}^{-2}$ & $\mathrm{p}<0.001, \mathrm{~F}=41.50, \mathrm{Fcrit}=2.39$ \\
Bulk Density & $0.96-1.74 \mathrm{~g} \cdot \mathrm{cm}^{-3}$ & $\mathrm{p}<0.05, \mathrm{~F}=2.77, \mathrm{Fcrit}=2.39$ \\
\hline
\end{tabular}

the maximum and minimum SOC stocks were recorded at S4 $(135.12 \pm 28.61$ $\left.\mathrm{kg} \cdot \mathrm{C} \cdot \mathrm{m}^{-2}\right)$ and $\mathrm{S} 3\left(16.75 \pm 3.83 \mathrm{~kg} \cdot \mathrm{C} \cdot \mathrm{m}^{-2}\right)$, respectively (Figure 4$)$.

\section{Discussion}

\subsection{Soil Characteristics}

Soil texture, soil bulk density and redox potential are the most important soil properties that affect the organic carbon concentrations and its stock in the Sundarbans mangrove soils. Lower bulk density results in a higher organic matter content. A lower level of bulk density was found in the Sundarbans soils except S4 due to litters and roots that serve as habitats for aquatic biota and thus consequent activities increase soil pore and macropore development (Eid et al., 2019). The bulk density of Sundarbans is lower than that of in Egypt (1.27 - 1.88 
Table 4. Concentration of five fractions of SOC $\left(\mu \mathrm{g} \cdot \mathrm{g}^{-1}\right)$ in studied areas.

\begin{tabular}{|c|c|c|c|c|c|c|}
\hline \multirow{2}{*}{ Soil } & \multirow{2}{*}{$\begin{array}{l}\text { Depth } \\
(\mathrm{cm})\end{array}$} & CWSC & HWSC & MLF & $\mathrm{MBC}$ & RF \\
\hline & & \multicolumn{5}{|c|}{ Concentration $\left(\mu \mathrm{g} \cdot \mathrm{g}^{-1}\right)$} \\
\hline \multirow{3}{*}{ S1 } & $0-15$ & $333.3 \pm 14.8$ & $608.0 \pm 26.4$ & $718.2 \pm 31.9$ & $456.23 \pm 53.2$ & $16,214.3 \pm 300$ \\
\hline & $15-50$ & $328.8 \pm 18.3$ & $556.8 \pm 17.4$ & $616.95 \pm 13.3$ & $365.86 \pm 27.6$ & $15,291.7 \pm 500$ \\
\hline & $50-100$ & $290.3 \pm 10.1$ & $480.4 \pm 12.8$ & $632.0 \pm 36 . .3$ & $245.7 \pm 26.3$ & $11,181.6 \pm 400$ \\
\hline \multirow{3}{*}{ S2 } & $0-15$ & $361.3 \pm 6.8$ & $589.5 \pm 21.7$ & $832.42 \pm 3.1$ & $405.43 \pm 36.5$ & $16,220.2 \pm 400$ \\
\hline & $15-50$ & $332.1 \pm 7.4$ & $544.7 \pm 7.0$ & $814.05 \pm 14.2$ & $386.54 \pm 32.2$ & $15,449.6 \pm 200$ \\
\hline & $50-100$ & $318.3 \pm 12.3$ & $548.8 \pm 9.6$ & $774.8 \pm 16.6$ & $303 . .0 \pm 21.6$ & $12,685.1 \pm 300$ \\
\hline \multirow{3}{*}{ S3 } & $0-15$ & $327.4 \pm 21.3$ & $526.4 \pm 26.4$ & $745.72 \pm 0.4$ & $476.38 \pm 45.1$ & $12,354.1 \pm 300$ \\
\hline & $15-50$ & $347.8 \pm 6.1$ & $404.7 \pm 65.7$ & $652.2 \pm 14.2$ & $265.94 \pm 16.8$ & $10,419.7 \pm 200$ \\
\hline & $50-100$ & $280.9 \pm 7.3$ & $280.0 \pm 11.2$ & $582.1 \pm 17.4$ & $231.5 \pm 10.2$ & $14,495.5 \pm 100$ \\
\hline \multirow{3}{*}{ S4 } & $0-15$ & $568.8 \pm 48.9$ & $646.7 \pm 17.0$ & $1515.37 \pm 12.3$ & $604.81 \pm 52.4$ & $67,064.3 \pm 400$ \\
\hline & $15-50$ & $320.4 \pm 7.8$ & $634.1 \pm 23.9$ & $1341.6 \pm 25.2$ & $408.64 \pm 23.4$ & $79,195.3 \pm 900$ \\
\hline & $50-100$ & $328.4 \pm 8.6$ & $558.0 \pm 15.4$ & $1020.1 \pm 22.1$ & $288.9 \pm 11.9$ & $82,744.6 \pm 300$ \\
\hline \multirow{3}{*}{ S5 } & $0-15$ & $352.82 \pm 13.9$ & $637.2 \pm 17.8$ & $864.82 \pm 17.2$ & $475.73 \pm 36.9$ & $15,219.5 \pm 500$ \\
\hline & $15-50$ & $321.17 \pm 10.0$ & $573.1 \pm 14.4$ & $822.82 \pm 6.4$ & $312.28 \pm 10.7$ & $15,910.6 \pm 600$ \\
\hline & $50-100$ & $274.7 \pm 17.6$ & $593.3 \pm 25.7$ & $745.8 \pm 30.6$ & $268.8 \pm 15.7$ & $18,477.4 \pm 200$ \\
\hline \multirow{3}{*}{ S6 } & $0-15$ & $333.93 \pm 7.6$ & $562.1 \pm 22.5$ & $1085.02 \pm 3.7$ & $469.69 \pm 22.5$ & $10,809.3 \pm 200$ \\
\hline & $15-50$ & $332.82 \pm 17.2$ & $515.3 \pm 43.9$ & $1125.07 \pm 16.9$ & $378.47 \pm 42.1$ & $12,468.3 \pm 700$ \\
\hline & $50-100$ & $301.2 \pm 8.4$ & $491.5 \pm 21.3$ & $856.9 \pm 16.1$ & $338.4 \pm 14.3$ & $13,132.0 \pm 400$ \\
\hline \multirow{3}{*}{ S7 } & $0-15$ & $334.52 \pm 10.9$ & $559.5 \pm 21.7$ & $1006.72 \pm 7.3$ & $407.42 \pm 50.5$ & $14,461.9 \pm 2200$ \\
\hline & $15-50$ & $310.2 \pm 15.4$ & $505.4 \pm 22.7$ & $810.82 \pm 2.7$ & $386.83 \pm 33.2$ & $13,196.8 \pm 900$ \\
\hline & $50-100$ & $284.5 \pm 11.1$ & $543.3 \pm 9.8$ & $918.7 \pm 31.2$ & $306.4 \pm 22.4$ & $16,227.1 \pm 200$ \\
\hline \multirow{3}{*}{ S8 } & $0-15$ & $335.85 \pm 10.5$ & $595.1 \pm 24.3$ & $1259.1 \pm 4.8$ & $443.63 \pm 42.8$ & $11,406.4 \pm 300$ \\
\hline & $15-50$ & $342.54 \pm 9.1$ & $552.2 \pm 21.0$ & $961.8 \pm 7.2$ & $318.85 \pm 12.4$ & $14,204.7 \pm 500$ \\
\hline & $50-100$ & $298.7 \pm 25.7$ & $512.3 \pm 6.6$ & $538.7 \pm 30.1$ & $306.1 \pm 21.6$ & $10,984.2 \pm 300$ \\
\hline \multirow{3}{*}{ S9 } & $0-15$ & $324.54 \pm 6.6$ & $568.8 \pm 13.3$ & $1425.15 \pm 4.9$ & $514.51 \pm 68.8$ & $11,987.0 \pm 500$ \\
\hline & $15-50$ & $301.34 \pm 14.4$ & $529.1 \pm 13.2$ & $1264.8 \pm 127.3$ & $478.26 \pm 71.6$ & $11,076.5 \pm 400$ \\
\hline & $50-100$ & $180.8 \pm 32.0$ & $381.1 \pm 44.7$ & $1010.9 \pm 98.3$ & $379.9 \pm 32.6$ & $9187.2 \pm 100$ \\
\hline \multirow{3}{*}{ S10 } & $0-15$ & $315.82 \pm 6.4$ & $480.3 \pm 10.9$ & $864.9 \pm 30.7$ & $365.35 \pm 19.2$ & $14,353.6 \pm 400$ \\
\hline & $15-50$ & $354.99 \pm 10.3$ & $424.8 \pm 26.7$ & $841.27 \pm 33.8$ & $345.91 \pm 41.1$ & $13,633.1 \pm 900$ \\
\hline & $50-100$ & $216.3 \pm 23.8$ & $356.1 \pm 36.1$ & $612.5 \pm 27.3$ & $313.4 \pm 18.6$ & $11,601.7 \pm 300$ \\
\hline
\end{tabular}

Table 5. Pearson correlation coefficient between different SOC fractions and soil properties.

\begin{tabular}{|c|c|c|c|c|c|}
\hline Fractions & CWSC & HWSC & MLF & MBC & RF \\
\hline HWSC & $0.574^{\star *}$ & & & & \\
\hline MLF & 0.248 & $0.480^{\star *}$ & & & \\
\hline $\mathrm{MBC}$ & $0.375^{\star}$ & $0.550^{\star *}$ & $0.689^{* *}$ & & \\
\hline RF & 0.220 & $0.377^{\star}$ & $0.455^{\star *}$ & 0.163 & \\
\hline TSOC & 0.277 & $0.388^{\star}$ & $0.470^{* *}$ & 0.178 & $1.000^{\star *}$ \\
\hline $\mathrm{BD}$ & -0.268 & 0.056 & 0.195 & -0.254 & $0.514^{\star *}$ \\
\hline $\mathrm{pH}$ & -0.189 & $-0.477^{\star}$ & $-0.766^{\star *}$ & $-0.525^{\star}$ & $-0.819^{* *}$ \\
\hline Clay & 0.060 & 0.402 & $0.900^{* *}$ & $0.696^{\star *}$ & $0.598^{\star \star}$ \\
\hline Eh & 0.171 & $0.537^{\star}$ & $0.647^{\star *}$ & $0.530^{*}$ & $0.909^{\star *}$ \\
\hline
\end{tabular}

${ }^{\star}$ Indicates 0.05 level of significance; ${ }^{* \star}$ Indicates 0.01 level of significance. 


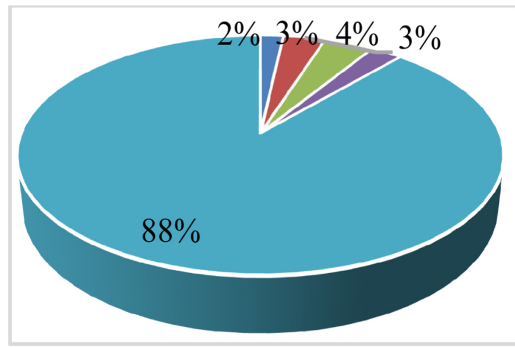

S1: $0-15 \mathrm{~cm}$

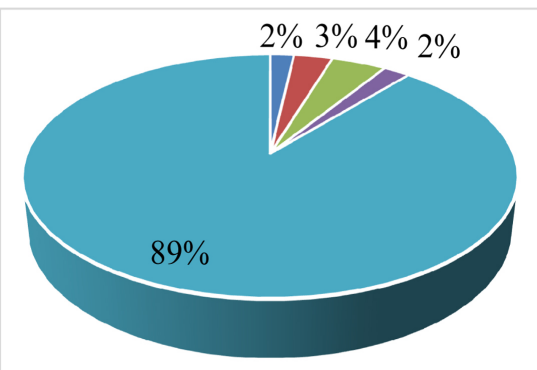

S2: $0-15 \mathrm{~cm}$

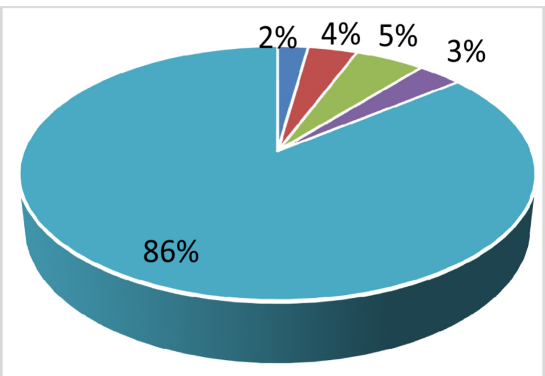

S3: $0-15 \mathrm{~cm}$

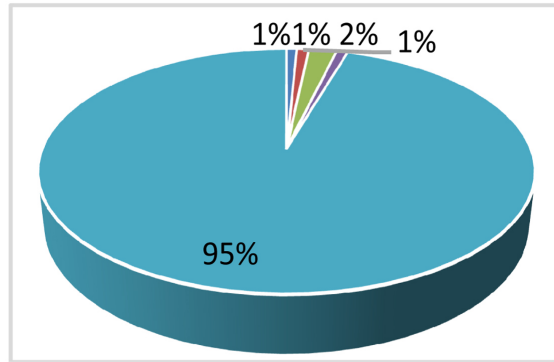

S4: 0-15 cm

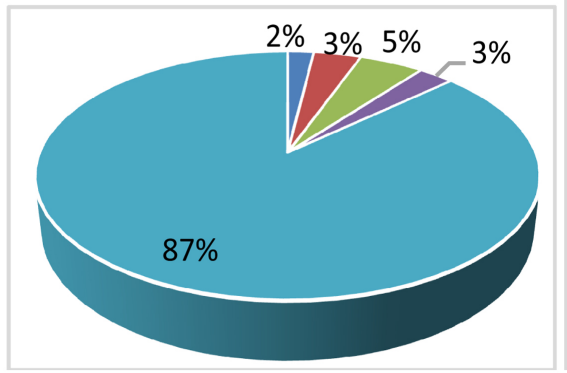

S5: 0-15 cm

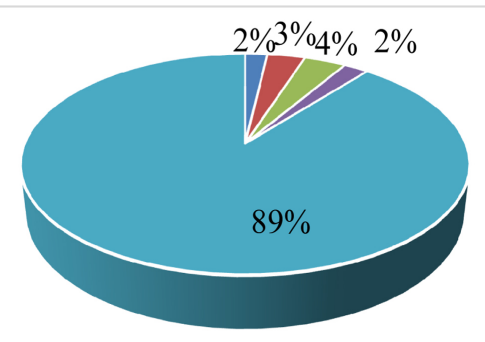

$\mathrm{S} 1: 15-50 \mathrm{~cm}$

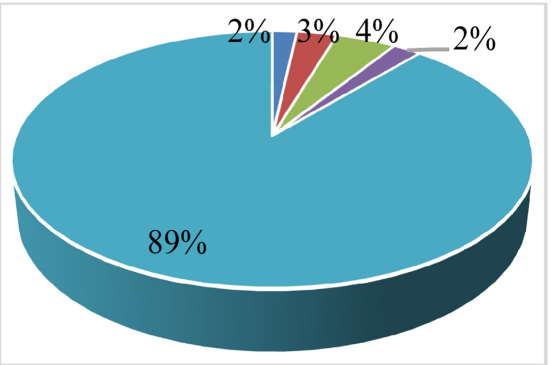

$\mathrm{S} 2: 15-50 \mathrm{~cm}$

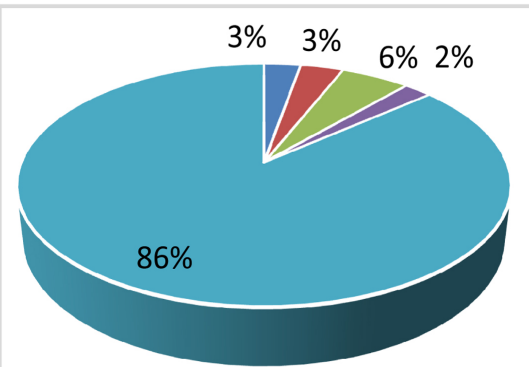

S3: $15-50 \mathrm{~cm}$

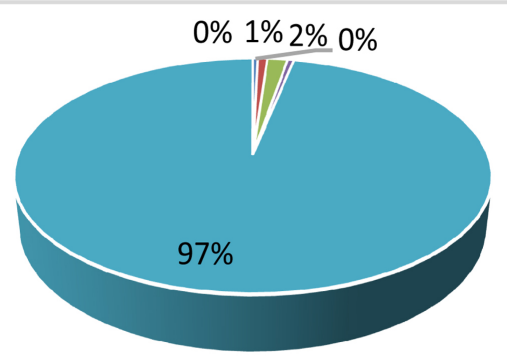

S4: $15-50 \mathrm{~cm}$

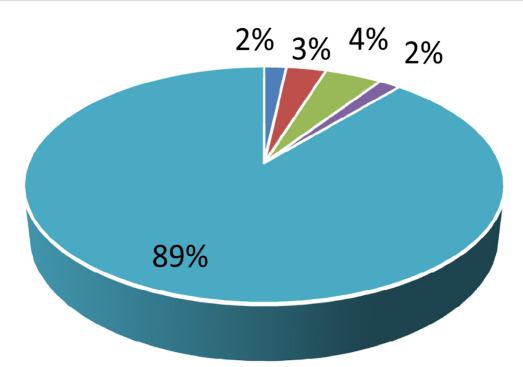

$\mathrm{S} 5: 15-50 \mathrm{~cm}$

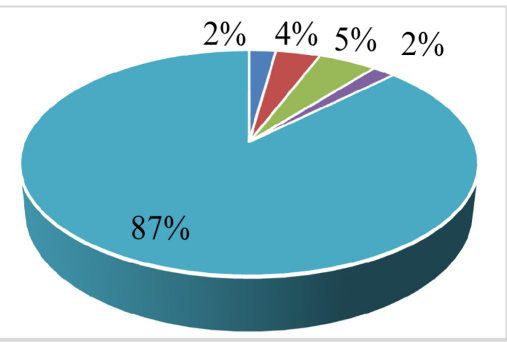

S1: $50-100 \mathrm{~cm}$

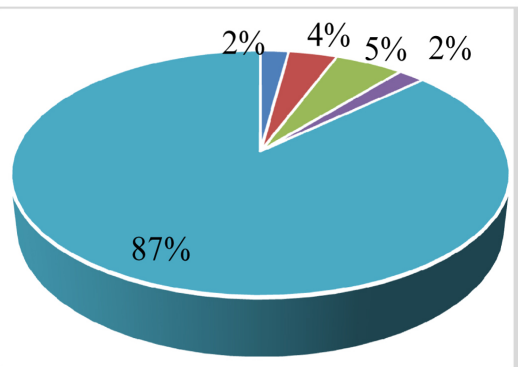

S2: $50-100 \mathrm{~cm}$

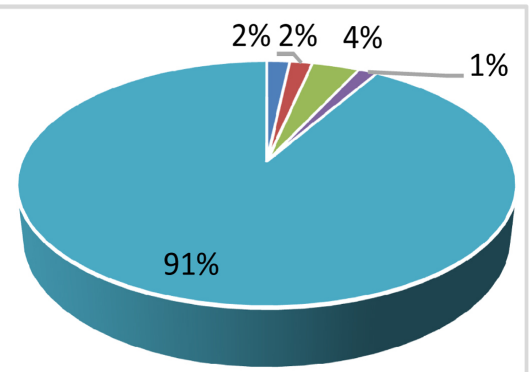

S3: $50-100 \mathrm{~cm}$

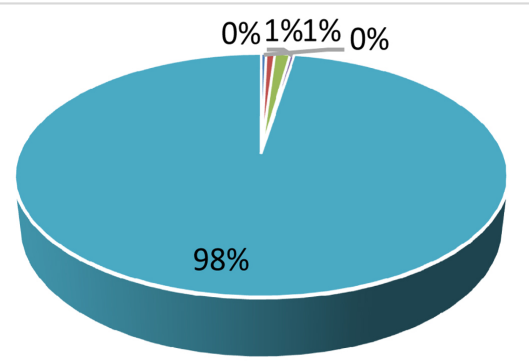

S4: $50-100 \mathrm{~cm}$

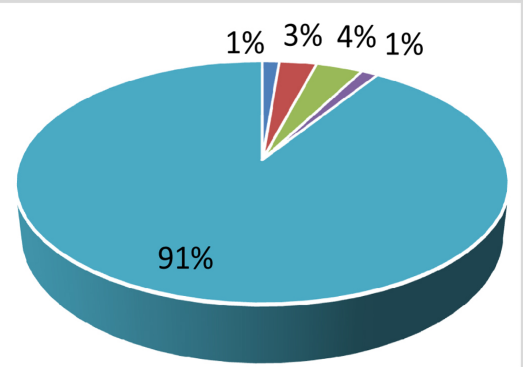

S5: $50-100 \mathrm{~cm}$ 
S. M. Akther et al.

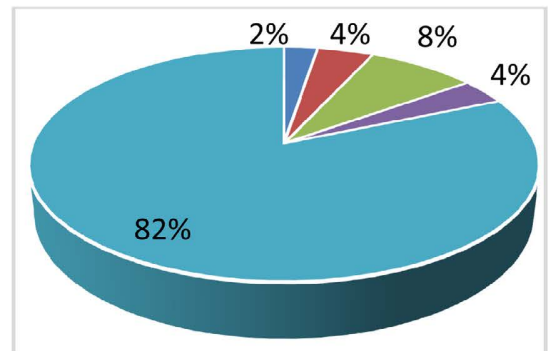

S6: 0-15 cm

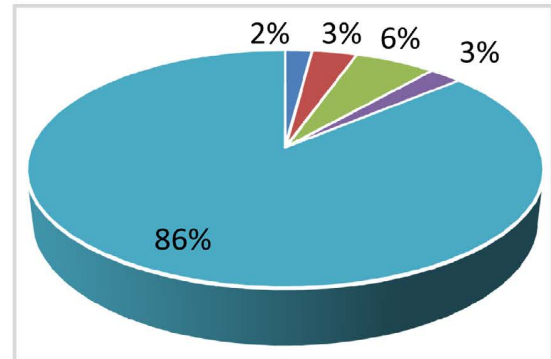

S7: 0-15 cm

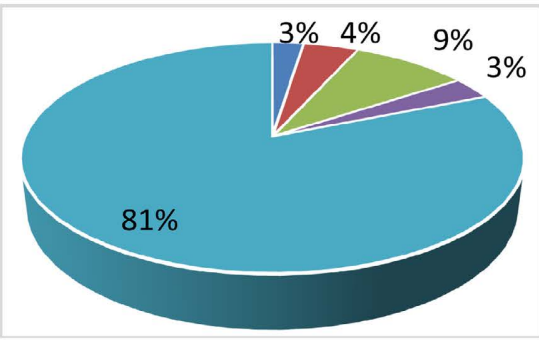

S8: 0-15 cm

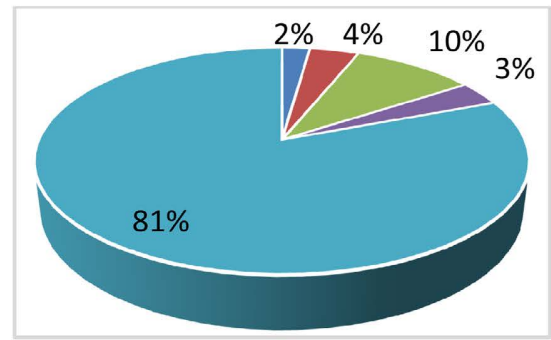

S9: 0-15 cm

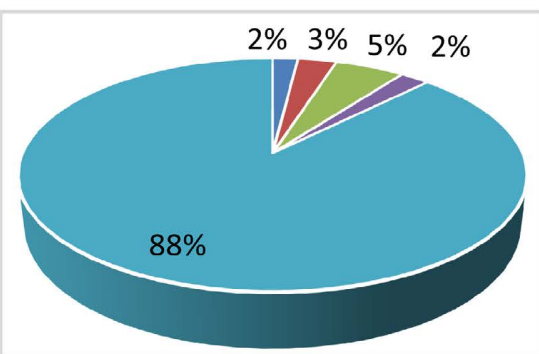

S10: 0-15 cm

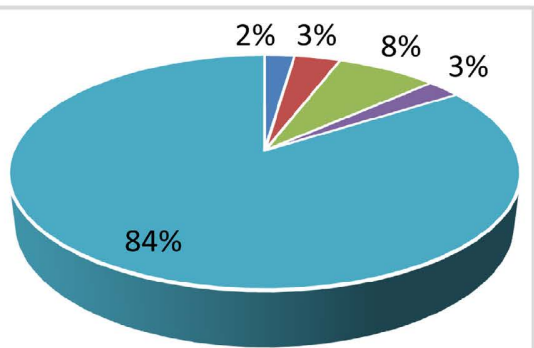

S6: $15-50 \mathrm{~cm}$

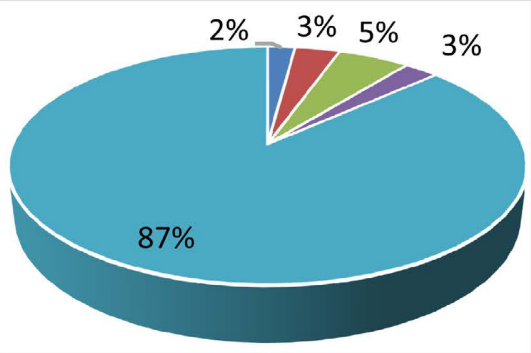

S7: $15-50 \mathrm{~cm}$

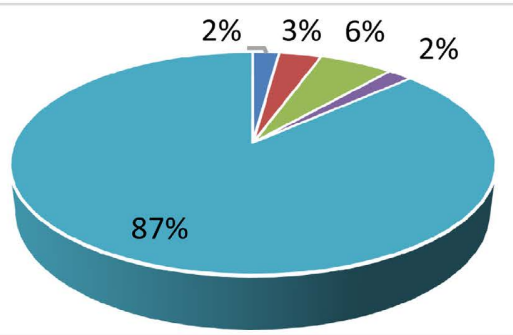

S8: $15-50 \mathrm{~cm}$

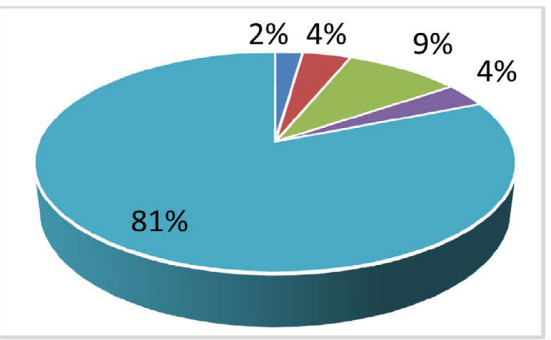

S9: $15-50 \mathrm{~cm}$

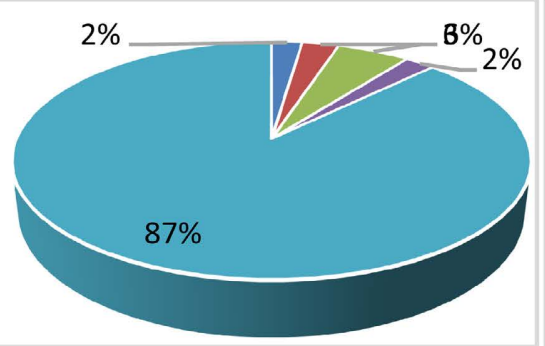

S10: $15-50 \mathrm{~cm}$

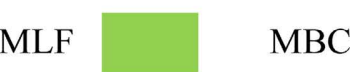

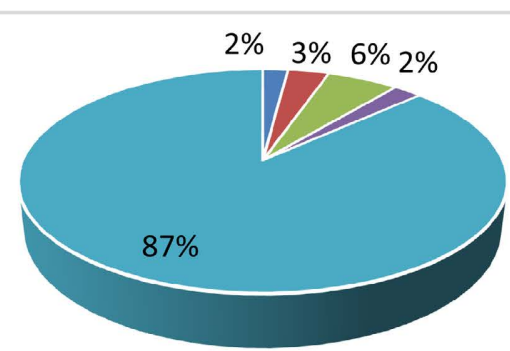

S6: $50-100 \mathrm{~cm}$

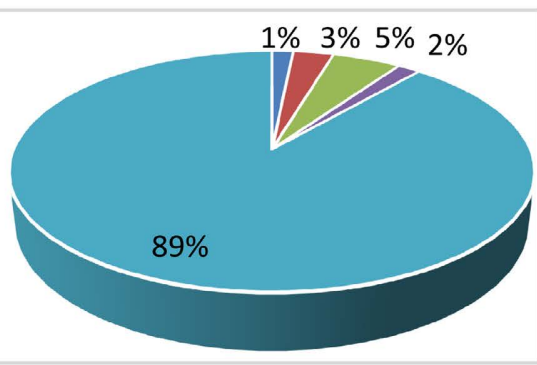

S7: $50-100 \mathrm{~cm}$

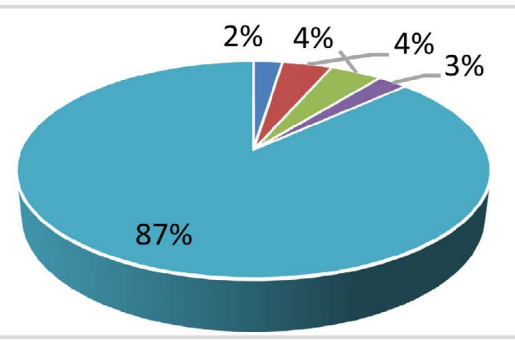

S8: $50-100 \mathrm{~cm}$

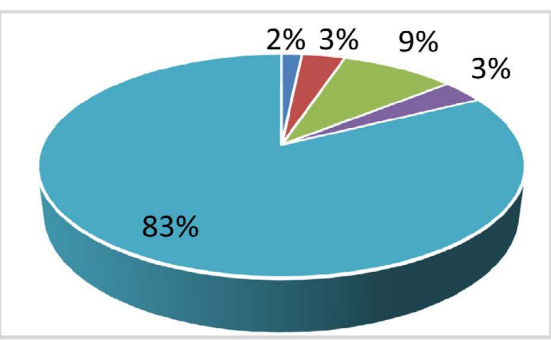

S9: $50-100 \mathrm{~cm}$

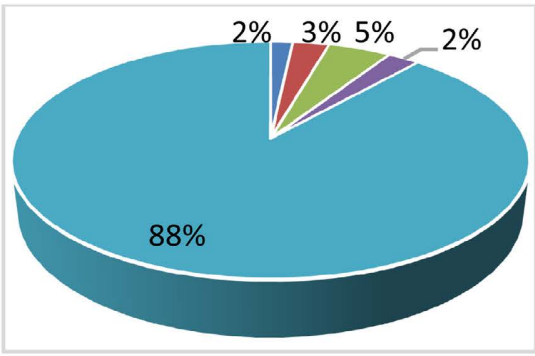

S10: $50-100 \mathrm{~cm}$

RF

Figure 2. Distribution of Carbon (\%) in different fractions of Sundarbans soils. 

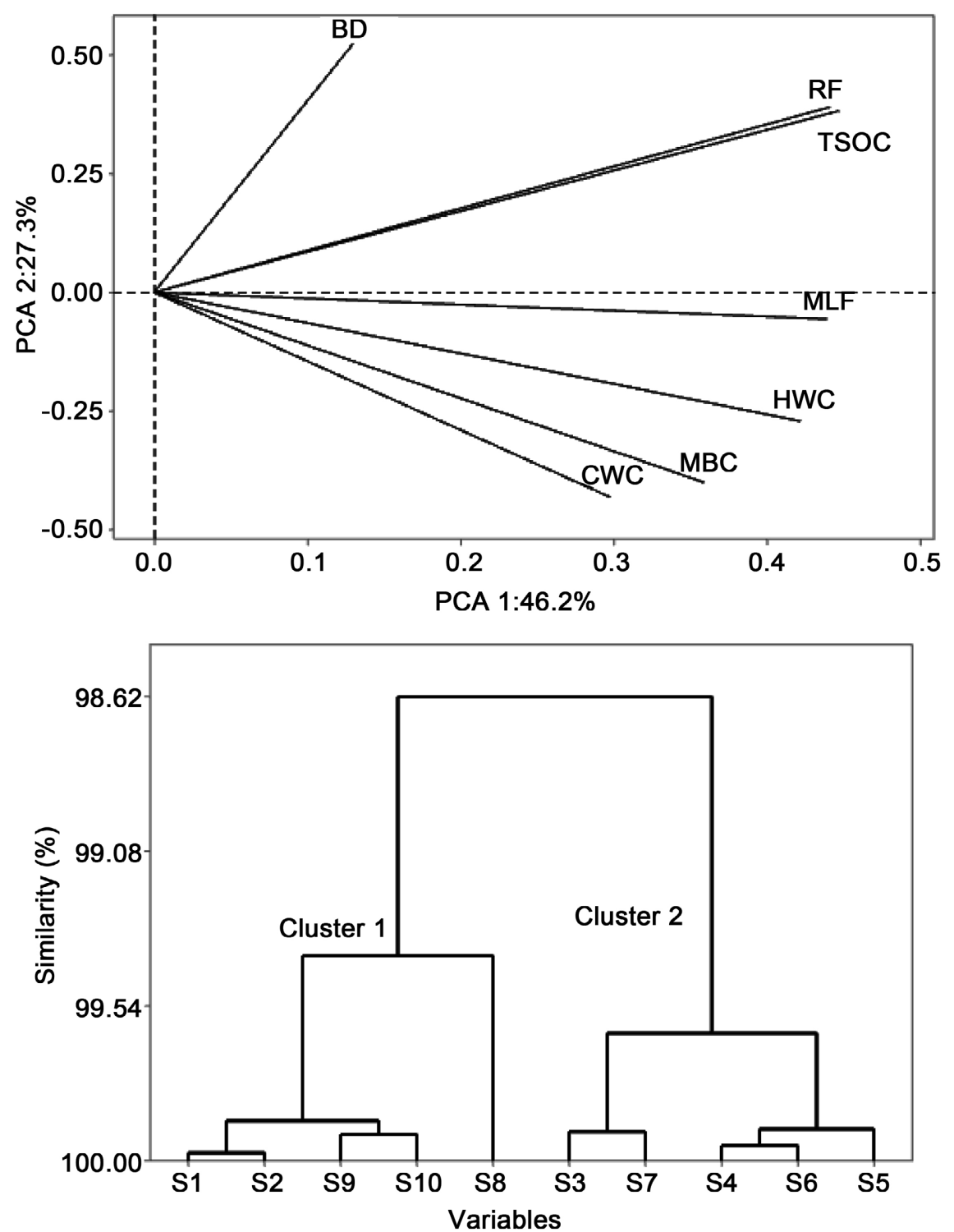

Figure 3. Principal Component Analysis (PCA) and Cluster Analysis of the five-carbon fractions and ten sampling locations of the Bangladesh Sundarbans.

$\mathrm{g} \cdot \mathrm{cm}^{-3}$ ) found by Eid and Shaltout (2016), higher than that of in Indonesia $\left(0.16-0.76 \mathrm{~g} \cdot \mathrm{cm}^{-3}\right)$ reported by Arifanti et al. (2019) and similar to $0.90-1.40$ $\mathrm{g} \cdot \mathrm{cm}^{-3}$ in Sri Lanka (Perera \& Amarasinghe, 2019). The main possible reason for this worldwide variability is differences in soil texture and organic matter decomposition rate.

The Sundarbans soils showed a higher SOC content than most of the mineral soils in Bangladesh. The current results were consistent with the results of Eid et al. (2019), Arifanti et al. (2019), Perera and Amarasinghe (2019), and Kauffman et al. (2018), who stated that mangroves have higher SOC sequestration capacity in comparison to other lands. In the current study, a trend of decreasing SOC with increasing depth was determined in this study, although some soils contained higher SOC in $15-50 \mathrm{~cm}$ depth (Table 2). 


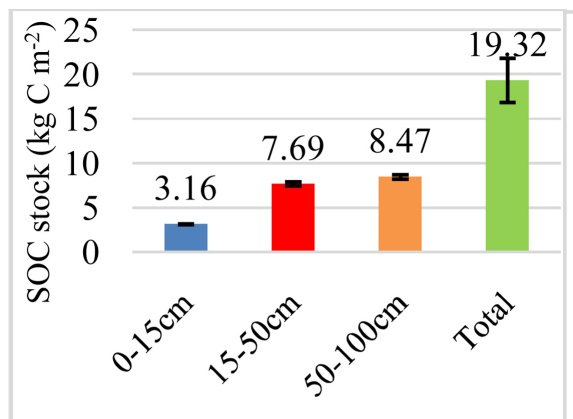

S1

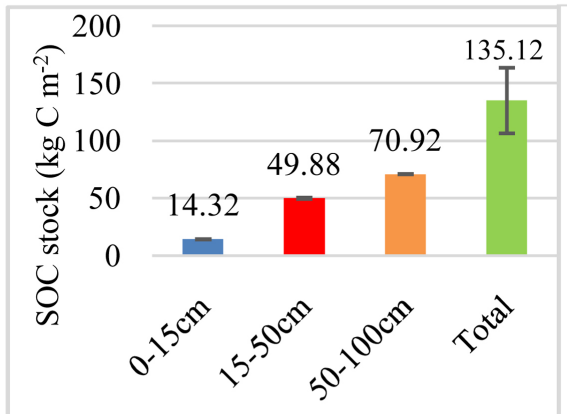

S4

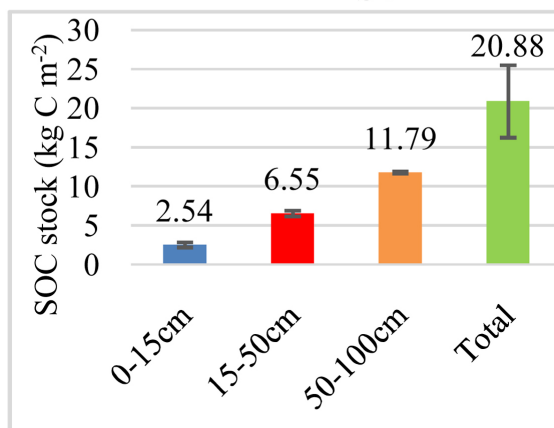

S7

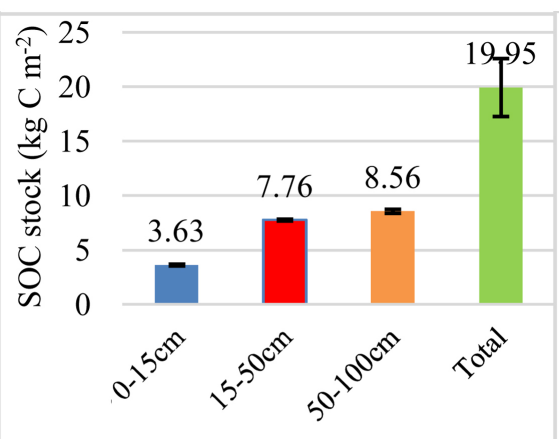

S2

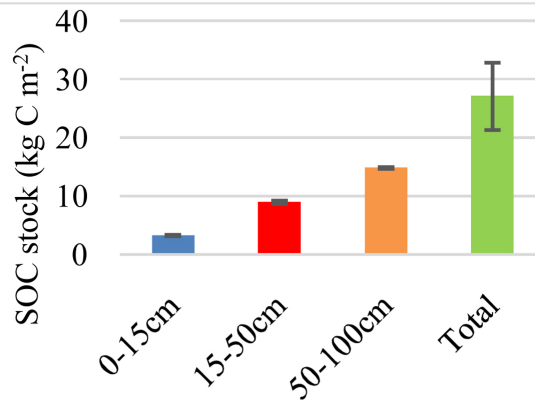

S5

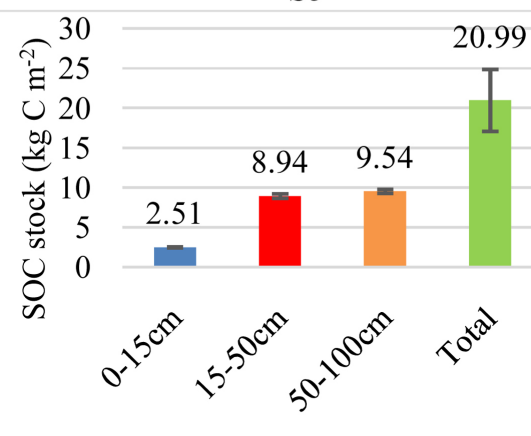

S8

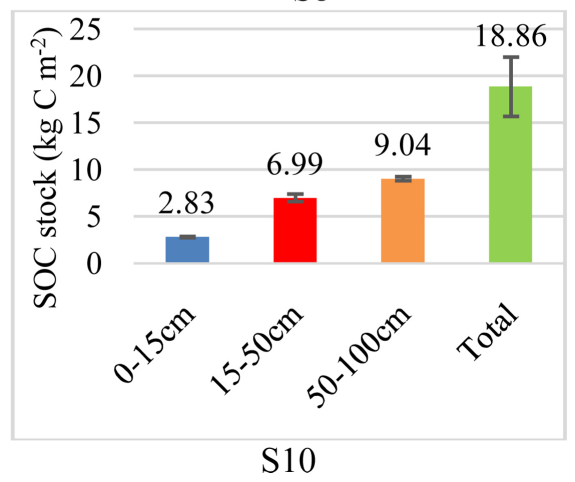

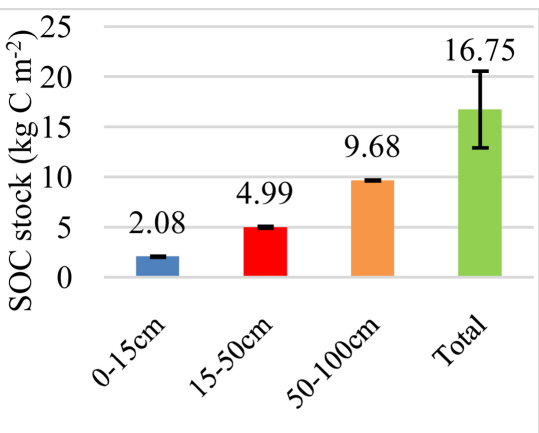

S3

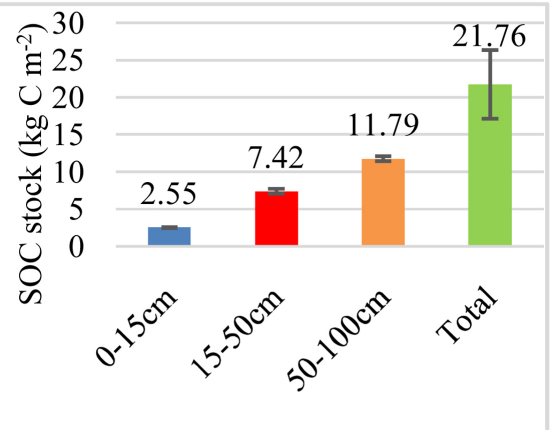

S6

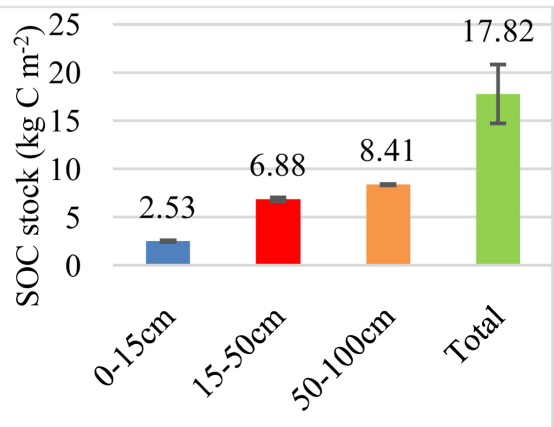

S9

\subsection{Fractionation of SOC}

Fractionation of soil organic carbon can give unique understandings into its distribution in the Sundarbans ecosystem and respond to greenhouse gas emissions mainly, $\mathrm{CO}_{2}$ and $\mathrm{CH}_{4}$. The cold-water soluble carbon (CWSC) fraction is considerably smaller than other carbon pools and constituted about only $\sim 0 \%$ to $3 \%$ 
of the total SOC (Figure 3). There was a decreasing trend of cold-water soluble $\mathrm{C}$ concentration from surface to $100 \mathrm{~cm}$, although some soils contain higher $\mathrm{C}$ in the $15-50 \mathrm{~cm}$ depth. The amount of CWSC varies regarding soil types, primarily depending on soil carbon content and microbial activity. A similar $\mathrm{C}$ concentration in CWSC (1\% to $1.25 \%$ ) fraction was reported in potato ecosystem, Maine, USA (Erich et al., 2012). However, several researchers described $0.1 \%$ to $0.4 \%$ CWSC in oven-dry soils (Provenzano et al., 2010) and about $1 \%$ in fieldmoist soils in the Arial Beel wetland of Bangladesh (Eva et al., 2018). It has been suggested that the CWSC being part of the highly labile C pool, may be susceptible to stress and perturbation in the soil-plant ecosystem (Doran \& Parkins, 1994) and, thus, might be a vital source of greenhouse gasses. On the contrary, the HWSC fraction was higher in all the studied soil than in the CWSC fraction. Partly extraction of non-humified organic material such as lignin, lignocellulose, and other carbohydrates by hot water may be possible for this higher $\mathrm{C}$ content in HWSC fraction (Hossain et al., 2020; Wang \& Wang, 2007). A similar HWSC carbon concentration $\left(361-865 \mu \mathrm{g} \cdot \mathrm{g}^{-1}\right)$ was reported by Eva et al. (2018) in the Arial Beel wetland, Bangladesh.

The moderately labile $\mathrm{C}$ fraction comprised a wide range (2\% to $10 \%$ ) of the total SOC and constituted a greater amount of C than CWSC and HWSC fractions. This fraction possibly represents carbon that can chemically sorb to clay surface and protected from decomposition (Hossain et al., 2020; Eva et al., 2018). Moderately labile fraction implies that this $\mathrm{C}$ fraction isn't readily available to microbes for decomposition and may have fewer impacts on climate change. Soil microbial biomass $\mathrm{C}$ is a determination of the carbon associated with the living components of the soil. Microorganisms are the main driving force of the $\mathrm{C}$ cycle that decompose soil organic matter, release $\mathrm{C}$ as $\mathrm{CO}_{2}$ in the atmosphere, and assimilate some $\mathrm{C}$ in their body mass (Sylvia et al., 2005). Microbial biomass C ranges from $1 \%$ to $5 \%$ of the total SOC but could be as much as $8 \%$ (Erich et al., 2012). This study found a range of about $0 \%$ to $4 \%$ of total SOC (Figure 2), well agreement with another study, 2\% - 5\% MBC in the Arial Beel wetland in Bangladesh (Eva et al., 2018). The MBC fraction gradually decreased from surface to substratum in all the soils. In this study, a higher amount of $\mathrm{C}$ was determined might be due to the presence of both aerobic and anaerobic microbes and very high organic matter in Sundarbans wetland. Besides, the distribution of dissolved organic carbon regulates the distribution of microbial biomass in soil (Zhang et al., 2006) because it is the principal energy source for microorganisms (Hofman et al., 2003; Haynes \& Francis, 1993).

A substantial quantity of carbon is predominately associated with the resistant fraction in the Sundarbans soils. The resistant C ranged from $9187.2 \pm 100$ to $82744.6 \pm 300 \mu \mathrm{g} \cdot \mathrm{g}^{-1}$, consisting of about $81 \%$ to $97 \%$ of the total SOC. Hossain et al. (2020) reported 75\% to $88 \%$ RF carbon in Arial Beel soils. Resistant carbon fraction (RF) can take centuries to decompose and is mostly unavailable to microbes because most of the $\mathrm{C}$ is stored as resistant pools in mangrove soils (Alongi, 
2012). Thus, this carbon fraction is sequestered into the soils of Bangladesh Sundarbans.

\subsection{Correlation Analysis}

The correlations between different carbon fractions and between carbon fractions and soil properties are presented in Table 5. Cold-Water soluble carbon showed a positive and significant relationship with HWSC and MBC, whereas the HWSC fraction significantly correlated with all the soil carbon fractions. This relationship implied that the content of labile carbon fractions influences both the mass and activity of microorganisms in the soil (Hoyle \& Murphy, 2006). The significant positive correlations between $C$ fractions suggested that $C$ pools are interdependent and need proper management plans to increase the $C$ pools in Sundarbans. This study found no significant relationship of CWSC and MBC fractions with total organic carbon (TOC). Also, the strong positive correlation of MLF with clay content supports the result of another research (Erich et al., 2012) that described the functional groups of clay minerals that control the pyrophosphate-extractable $\mathrm{C}$. This fraction comprises molecules soluble via a ligand exchange reaction that detaches $\mathrm{Fe}$ and $\mathrm{Al}$ cations. This study also indicated that anaerobic condition is the main regulatory factor of mangrove wetlands.

The principal component analysis (PCA) also emphasized a highly positive relationship among five $\mathrm{C}$ pools in Sundarbans (Figure 3 ) and the two components (PCA 1 and PCA 2) together explained a total of $73.5 \%$ data variability in the studied areas. Multivariate analysis depicted $99.39 \%$ similarities among S1, S2, S9, S10, and S8 in cluster 1, while S3, S7, S4, S6, and S5 showed 99.62\% similarities in cluster 2 (Figure 3). Since S1 and S2 samples were collected from comparatively closer locations, they showed maximum similarities. Contrary, S3-S7 showed similar soil properties and carbon fractions because these are the nearest to the Bay of Bengal coast.

\subsection{Storage of Organic Carbon in the Sundarbans Mangrove Soils}

The stock of SOC in surface $(0-15 \mathrm{~cm})$, subsurface $(15-50 \mathrm{~cm})$, substratum $(50$ $100 \mathrm{~cm}$ ), and total stock in $1 \mathrm{~m}$ soil profile of the Sundarbans mangrove soils are illustrated in Figure 4. SOC stock in the Sundarbans soils was determined in $1 \mathrm{~m}$ depth because substantial SOC may accumulate in lower soil layers (Lal, 2008). Although microbial activity is the principal regulatory agent of SOC stabilization and mineralization, the dynamics, nature, origin, and properties of deeper soil carbon in changing global environment are largely unknown. Thus, this experiment aimed to understand the carbon dynamics of up to $100 \mathrm{~cm}$ soil profile in Sundarbans mangrove.

Results showed that SOC stock in Sundarbans mangrove soils varied considerably and ranged from $16.75 \pm 3.83$ to $135.12 \pm 28.61 \mathrm{~kg} \cdot \mathrm{C} \cdot \mathrm{m}^{-2}$ in $1 \mathrm{~m}$ soil profile. The maximum SOC stocks in all the three studied depths were determined 
in S4 (14.32, 49.88, and $70.93 \mathrm{~kg} \cdot \mathrm{C} \cdot \mathrm{m}^{-2}$, upper to lower respectively) possibly due to higher burial of organic matter by natural calamities. In contrast, the minimum SOC stocks in the three studied depths were found in S3, S7, and S9, respectively. The soils of $50-100 \mathrm{~cm}$ contained more carbon than the other two layers because the bulk density and thickness of this lower layer are higher (50 $\mathrm{m})$ than the surface $(15 \mathrm{~cm})$ and subsurface $(35 \mathrm{~cm})$. Also, more than 8 times higher SOC storage was determined in S4 than S3. The subsurface and substratum $\mathrm{C}$ sources are mainly root residues, water transportation of particulate and dissolved organic $C$ from the soil surface, and bioturbation (Rasse et al., 2006). Comparing with the other studies globally, the SOC stocks in the Sundarbans mangrove were higher than the described values in Saudi Arabia (19.9 to 29.2 $\left.\mathrm{kg} \cdot \mathrm{C} \cdot \mathrm{m}^{-2}\right)$, Vietnam $\left(5.8 \mathrm{~kg} \cdot \mathrm{C} \cdot \mathrm{m}^{-2}\right)$, Egypt $\left(8.5 \mathrm{~kg} \cdot \mathrm{C} \cdot \mathrm{m}^{-2}\right)$, and New Zealand $(6.9$ $\mathrm{kg} \cdot \mathrm{C} \cdot \mathrm{m}^{-2}$ ) reported by Eid et al. (2019), Grellier et al. (2017), Eid and Shaltout, (2016), and Pérez et al. (2017), respectively. On the other hand, the results were lower than that of mangroves in Micronesia $\left(73-215 \mathrm{~kg} \cdot \mathrm{C} \cdot \mathrm{m}^{-2}\right.$ ) by Kauffman et al. (2011), Sri Lanka (average $59.0 \mathrm{~kg} \cdot \mathrm{C} \cdot \mathrm{m}^{-2}$ ) by Perera and Amarasinghe (2019), and Indonesia (average $68.8 \mathrm{~kg} \cdot \mathrm{C} \cdot \mathrm{m}^{-2}$ ) by Kusumaningtyas et al. (2019). However, the role of Sundarbans mangrove forest in mitigating global climate change might be more significant than that of conventionally ascribed.

\section{Conclusion}

Soil organic carbon is a vital component of the Sundarbans soils that significantly impacts the proper functioning of its ecosystem. The SOC fractionation study showed that the Cold-Water soluble carbon (CWSC) fraction was considerably smaller than other fractions that primarily depend on total SOC content and soil microbial activities. The moderately labile (MLF) and hot-water soluble (HWSC) fractions were the second and third most dominant fractions, included root exudates, clay bound $\mathrm{C}$, soluble amino acids and carbohydrates. The SOC was mainly associated with the resistant fraction of about $81 \%$ to $97 \%$ of total SOC. Resistant carbon fraction (RF) takes centuries to decompose and is largely unavailable to microbes. Moreover, these five SOC pools were significantly correlated with two or more other fractions and thus, changes in one pool can cause carbon imbalance in the Sundarbans ecosystem. Besides, Sundarbans soils contained a high SOC stock in the studied soil profiles. Therefore, this study revealed that Sundarbans mangrove soils are a good carbon sink and suggested that sustainable management of the Sundarbans mangrove reserve would increase the SOC storage and contribute to climate change mitigation.

\section{Acknowledgements}

We are grateful to the partial financial support of the Ministry of Science and Technology, Government of Bangladesh, for MS thesis research (National Science and Technology Fellowship: 2019-2020; Merit No. 126). We are also thankful to 
the Centre for Advanced Research in Science (CARS), University of Dhaka, for providing carbon analysis facilities.

\section{Authors' Contributions}

Sayada Momotaz Akther and Zakia Parveen conceived and planned the experiments. Sayada Momotaz Akther and Md Mahfuz Islam carried out the lab experiment, performed data analysis, and wrote the manuscript with inputs from other authors. Md Faruque Hossain contributed to manuscript preparation. Zakia Parveen supervised this research.

\section{Submission Declaration and Verification}

This paper has not been published previously, and it is not under consideration for publication elsewhere. All the responsible authors approve its publication. If this paper is accepted, it will not be published elsewhere in the same form, in English or any other language, including electronically, without the written consent of the copyright holder.

\section{Funding}

This research did not receive any specific grant from funding agencies in the public, commercial, or not-for-profit sectors.

\section{Declaration of Interests}

The authors declare that they have no known competing financial interests or personal relationships that could have appeared to influence the work reported in this paper.

\section{References}

Alongi, D. M. (2002). Present State and Future of the World's Mangrove Forests. Environmental Conservation, 29, 331-349.

Alongi, D. M. (2008). Mangrove Forests: Resilience, Protection from Tsunamis, and Responses to Global Climate Change. Estuarine, Coastal and Shelf Science, 76, 1-13. https://doi.org/10.1016/j.ecss.2007.08.024

Alongi, D. M. (2012). Carbon Sequestration in Mangrove Forests. Carbon Management, 3, 313-322. https://doi.org/10.4155/cmt.12.20

Arifanti, V. B., Kauffman, J. B., Hadriyanto, D., Murdiyarso, D., \& Diana, R. (2019). Carbon Dynamics and Land Use Carbon Footprints in Mangrove-Converted Aquaculture: The Case of the Mahakam Delta, Indonesia. Forest Ecology and Management, 432, 17-29. https://doi.org/10.1016/j.foreco.2018.08.047

Blake, G. R. (1965). Bulk Density. In C. A. Black et al. (Eds.), Methods of Soil Analysis. Part 1. Physical and Mineralogical Properties, Including Statistics of Measurement and Sampling. Agronomy Series 9 (pp. 374-390). American Society of Agronomy, Inc.

Bouyoucos, G. J. (1936). Directions for Making Mechanical Analysis of Soils by the Hydrometer Method. Soil Science, 42, 225-230.

https://doi.org/10.1097/00010694-193609000-00007 
Bridgham, S. D., Megonigal, J. P., Keller, J. K., Bliss, N. B., \& Trettin, C. (2006). The Carbon Balance of North American Wetlands. Wetlands, 26, 889-916.

Donato, D. C., Kauffman, J. B., Murdiyarso, D., Kurnianto, S., \& Stidham, M. (2011). Mangroves among the Most Carbon-Rich Forests in the Tropics. Nature Geoscience, 4, 293-297. https://doi.org/10.1038/ngeo1123

Doran, J. W., \& Parkins, T. B. (1994). Defining and Assessing Soil Quality. In J. W. Doran, D. C. Coleman, D. F. Bezdicek, B. A., Stewart (Eds.), Defining Soil Quality for a Sustainable Environment (pp. 3-21). Soil Science Society America. https://doi.org/10.2136/sssaspecpub35.c1

Duke, N. C., Meynecke, J. O., Dittmann, S., Ellison, A. M., Anger, K., Berger, U., Cannicci, S., Diele, K., Ewel, K. C., Field, C. D., Koedam, N., Lee, S. Y., Marchand, C., Nordhaus, I., \& Dahdouh-Guebas, F. (2007). A World without Mangroves? Science, 317, 41-42. https://doi.org/10.1126/science.317.5834.41b

Eid, E. M., \& Shaltout, K. H. (2016). Distribution of Soil Organic Carbon in the Mangrove Avicennia marina (Forssk.) Vierh. along the Egyptian Red Sea Coast. Regional Studies in Marine Science, 3, 76-82. https://doi.org/10.1016/j.rsma.2015.05.006

Eid, E. M., Arshad M., Shaltout, K. H., El-Sheikh, M. A., Alfarhan, A. H., Picó, Y., \& Barcelo, D. (2019). Effect of the Conversion of Mangroves into Shrimp Farms on Carbon Stock in the Sediment along the Southern Red Sea Coast, Saudi Arabia. Environmental Research, 176, Article ID: 108536. https://doi.org/10.1016/j.envres.2019.108536

Erich, M. S., Plante, A. F., Ferandez, J. M., Mallory, E. B., \& Ohno, T. (2012). Effects of Profile Depth and Management on the Composition of Labile and Total Organic Matter. Soil Science Society of America Journal, 76, 408-419.

Eva, M. A., Piash, M. I., Hossain, M. F., \& Parveen, Z. (2018). Fractionation of Organic Carbon in Arial Beel Wetland Soils of Bangladesh. American Journal of Environmental Science, 14, 86-94.

Ghani, A., Dexer, M., \& Perrott, K. W. (2003). Hot-Water Extractable Carbon in Soils: A Sensitive Measurement for Determining Impacts of Fertilization, Grazing and Cultivation. Soil Biology and Biochemistry, 35, 1231-1243. https://doi.org/10.1016/S0038-0717(03)00186-X

Gregorich, E. G., Beare, M. H., Stoklas, U., \& St-Georges, P. (2003). Biodegradability of Soluble Organic Matter in Maize Cropped Soils. Geoderma, 113, 237-252. https://doi.org/10.1016/S0016-7061(02)00363-4

Grellier, S., Janeau, J. L., Nhon, D. H., Cuc, N. T. K., Quynh, L. T. P., Thao, P. T. T., Trang, T. T. N., \& Marchand, C. (2017). Changes in Soil Characteristics and C Dynamics after Mangrove Clearing (Vietnam). Science of the Total Environment, 593-594, 654-663. https://doi.org/10.1016/j.scitotenv.2017.03.204

Haynes, R. J., \& Francis, G. S. (1993). Changes in Microbial Biomass C, Soil Carbohydrate Composition and Aggregate Stability Induced by Growth of Selected Crop and Forage Species under Field Conditions. Journal of Soil Science, 44, 665-675.

Hofman, J., Bezchlebova, J., Dusek, L., Dolezal, L., Holoubek, I., Andel, P., Ansorgova, A., \& Maly, S. (2003). Novel Approach to Monitoring of the Soil Biological Quality. Environment International, 28, 771-778. https://doi.org/10.1016/S0160-4120(02)00068-5

Hossain, M. F., Chen, W., \& Zhang, Y. (2015). Bulk Density of Mineral and Organic Soils in the Canada's Arctic and Sub-Arctic. Information Processing in Agriculture, 2, 183-190. https://doi.org/10.1016/j.inpa.2015.09.001

Hossain, M. F., Maksud Kamal, A. S. M., Ahmed, S. M., Eva, M. A., \& Parveen, Z. (2020). Soil Organic Carbon Pool and Its Storage in Wetland Soils of Bangladesh. American 
Journal of Environmental Science, 16, 55-67.

Hossain, M. F., Zhang, Y., Chen, W., Wang, J., \& Pavlic, G. (2007). Soil Organic Carbon Content in Northern Canada: A Database of Field Measurements and Its Analysis. Canadian Journal Soil Science, 87, 259-268. https://doi.org/10.4141/S06-029

Houghton, R. A. (2007). Balancing the Global Carbon Budget. Annual Review of Earth and Planetary Sciences, 35, 313-347.

https://doi.org/10.1146/annurev.earth.35.031306.140057

Hoyle, F. C., \& Murphy, D. V. (2006). Seasonal Changes in Microbial Function and Diversity Associated with Stubble Retention versus Burning. Australian Journal of Soil Research, 44, 407-423. https://doi.org/10.1071/SR05183

Hussain, Z., \& Acharya, G. (1994). Mangrove of the Sundarbans, Volume Two: Bangladesh. IUCN Southeast Asia Regional Office.

Kauffman, J. B., Bernardino, A. F., Ferreira, T. O., Bolton, N. W., Gomes, L. E. O., \& Nobrega, G. N. (2018). Shrimp Ponds Lead to Massive Loss of Soil Carbon and Greenhouse Gas Emissions in Northeastern Brazilian Mangroves. Ecology and Evolution, 8, 5530-5540.

Kauffman, J. B., Heider, C., Cole, T. G., Dwire, K. A., \& Donato, D. (2011). Ecosystem Carbon Stocks of Micronesian Mangrove Forests. Wetlands, 31, 343-352. https://doi.org/10.1007/s13157-011-0148-9

Koshiba, S., Besebes, M., Soaladaob, K., Isechal, A. L., Victor, S., \& Golbuu, Y. (2013). Palau's Taro Fields and Mangroves Protect the Coral Reefs by Trapping Eroded Fine Sediment. Wetlands Ecology and Management, 21, 157-164.

https://doi.org/10.1007/s11273-013-9288-4

Kusumaningtyas, M. A., Hutahaean A. A., Fischer, H. W., Fischer, Pérez-Mayo, M., Ransby, D., \& Jennerjahn, T. C. (2019). Variability in the Organic Carbon Stocks, Sources, and Accumulation Rates of Indonesian Mangrove Ecosystems. Estuarine, Coastal and Shelf Science, 218, 310-323. https://doi.org/10.1016/j.ecss.2018.12.007

Lal, R. (2008). Carbon Sequestration. Philosophical Transactions of the Royal Society B, 363, 815-830. https://doi.org/10.1098/rstb.2007.2185

Meersmans, J., De Ridder, F., Canters, F., De Baets, S., \& Van Molle, M. (2008). A Multiple Regression Approach to Assess the Spatial Distribution of Soil Organic Carbon (SOC) at the Regional Scale (Flanders, Belgium). Geoderma, 143, 1-13. https://doi.org/10.1016/j.geoderma.2007.08.025

Mitsch, W. J., Bernal, B., Nahlik, A. M., Mander, U., Zhang, L., Anderson, C. J., Jorgensen, S. E., \& Brix, H. (2013). Wetlands, Carbon and Climate Change. Landscape Ecology, 28, 583-597. https://doi.org/10.1007/s10980-012-9758-8

Murdiyarso, D., Donato, D., Kauffman, J. B., Stidham, M., \& Kanninen, M. (2009). Carbon Storage in Mangrove and Peatland Ecosystems: A Preliminary Account from Plots in Indonesia. Center for International Forest Research, $37 \mathrm{p}$.

Murdiyarso, D., Purbopuspito, J., Kauffman, J. B., Warren, M. W., Sasmito, S. D., Donato, D. C., Manuri, S., Krisnawati, H., Taberima, S., \& Kurnianto, S. (2015). The Potential of Indonesian Mangrove Forests for Global Climate Change Mitigation. Nature Climate Change, 5, 1089-1092. https://doi.org/10.1038/nclimate2734

Oelkers, E. H., \& Cole, D. R. (2008). Carbon Dioxide Sequestration: A Solution to the Global Problem. Elements, 4, 305-310. https://doi.org/10.2113/gselements.4.5.305

Perera, K. A. R. S., \& Amarasinghe, M. D. (2019). Carbon Sequestration Capacity of Mangrove Soils in Micro Tidal Estuaries and Lagoons: A Case Study from Sri Lanka. Geoderma, 347, 80-89. https://doi.org/10.1016/j.geoderma.2019.03.041 
Pérez, A., Machado, W., Gutierrez, D., Stokes, D., Sanders, L., Smoak, J. M., Santos, I., \& Sanders, C. J. (2017). Changes in Organic Carbon Accumulation Driven by Mangrove Expansion and Deforestation in a New Zealand Estuary. Estuarine, Coastal and Shelf Science, 192, 108-116. https://doi.org/10.1016/j.ecss.2017.05.009

Pinheiro, E. F. M., Pereira, M. G., Anjos, L. H. C., \& Machado, P. L. O. A. (2004). Fracionamento densimétrico da material orgânica do solo sob diferentes sistemas de manejo e cobertura vegetal em Paty do Alferes. Revista Brasileira de Ciência do Solo, 28, 731-37. https://doi.org/10.1590/S0100-06832004000400013

Provenzano, M. R., Caricasole, P., Brunetti, G., \& Senesi, N. (2010). Dissolved Organic Matter Extracted with Water and a Saline Solution from Different Soil Profiles. Soil Science, 175, 255-262. https://doi.org/10.1097/SS.0b013e3181e457a6

Rasse, D. P., Mulder, J., Moni, C. \& Chenu, C. (2006). Carbon Turnover Kinetics with Depth in a French Loamy Soil. Soil Science Society of America Journal, 70, 2097-2105. https://doi.org/10.2136/sssaj2006.0056

Schnitzer, M., \& Schuppli, P. (1989). Method for the Sequential Extraction of Organic Matter from Soils and Soil Fractions. Soil Science Society of America Journal, 53, 1418-1424. https://doi.org/10.2136/sssaj1989.03615995005300050019x

Siddiqi, N. A. (2001). Mangroves of Bangladesh Sundarbans and Accretion Areas. In D. D. L. Lecerda (Ed.), Mangrove Ecosystems Function and Management (pp. 142-292). Springer-Verlag.

Spaulding, M., Kainuma, M., \& Collins, L. (2010). World Atlas of Mangroves. Earthscan.

Sylvia, D. M., Fuhrmann, J. J., Hartel, P. G., \& Zuberer, D. A. (2005). Principles and Applications of Soil Microbiology (2nd ed.). Prentice Hall.

Twilley, R. R., Chen, R. H., \& Hargis, T. (1992). Carbon Sinks in Mangroves and Their Implications to Carbon Budget of Tropical Coastal Ecosystems. Water, Air, and Soil Pollution, 64, 265-288. https://doi.org/10.1007/BF00477106

Vance, E. D., Brookes, P. C., \& Jenkinson, D. S. (1987). An Extraction Method for Measuring Soil Microbial Biomass Carbon. Soil Biology and Biochemistry, 19, 703-707. https://doi.org/10.1016/0038-0717(87)90052-6

Walkley, A., \& Black, I. A. (1934). An Examination of the Degtjareff Method for Determining Soil Organic Matter and a Proposed Modification of the Chromic Acid Titration Method. Soil Science, 37, 29-38. https://doi.org/10.1097/00010694-193401000-00003

Wang, Q. K., \& Wang, S. L. (2007). Soil Organic Matter under Different Forest Types in Southern China. Geoderma, 142, 349-356. https://doi.org/10.1016/j.geoderma.2007.09.006

Wattel-Koekkoek, E. J. W., van Genuchten, P. P. L., Buurman, P., \& van Lagen, B. (2001). Amount and Composition of Clay-Associated Soil Organic Matter in a Range of Kaolinitic and Smectitic Soils. Geoderma, 99, 27-49. https://doi.org/10.1016/S0016-7061(00)00062-8

Zhang, J. B., Song, C. C., \& Yang, W. Y. (2006). Land Use Effects on the Distribution of Labile Organic Carbon Fractions through Soil Profiles. Soil Science Society America Journal, 70, 660-667. 